http://dx.doi.org/10.35381/e.k.v3i6.829

\title{
Docencia universitaria y metodologías activas: una propuesta para generar aprendizaje significativo
}

\section{University teaching and active methodologies: a proposal to generate meaningful learning}

\author{
Luis Miguel Ormaza-Ulloa \\ lormazau@ucacue.edu.ec \\ Universidad Católica de Cuenca, Azogues \\ Ecuador \\ https://orcid.org/0000-0003-1964-8651 \\ Darwin Gabriel Garcia-Herrera \\ dggarciah@ucacue.edu.ec \\ Universidad Católica de Cuenca, Azogues \\ Ecuador \\ https://orcid.org/0000-0001-6813-8100 \\ Juan Carlos Erazo-Álvarez \\ jcerazo@ucacue.edu.ec \\ Universidad Católica de Cuenca, Cuenca \\ Ecuador \\ https://orcid.org/0000-0001-6480-2270 \\ Cecilia Ivonne Narváez-Zurita \\ inarvaez@ucacue.edu.ec \\ Universidad Católica de Cuenca, Cuenca \\ Ecuador \\ https://orcid.org/0000-0002-7437-9880
}

Recepción: 08 abril 2020

Revisado: 16 de mayo 2020

Aprobación: 15 junio 2020

Publicación: 1 de julio 2020 


\title{
RESUMEN
}

La investigación tuvo como objetivo analizar el uso de metodologías activas por parte de docentes universitarios de las ciencias médicas con la finalidad de generar un aprendizaje significativo en los estudiantes. Mediante un tipo descriptiva correlacional en un universo de 90 docentes de la Carrera de Medicina de la Universidad Católica de Cuenca. Los resultados indican que la frecuencia del uso de metodologías activas en clases con las principales dificultades observadas en sus alumnos no se encontró relación estadísticamente significativa, a la vez se verifico las variables de preparación académica en pedagogía en los docentes, denotando valores superiores de quienes no tenían dicha preparación, de la misma forma resulto sobresaliente el uso del Trabajo Colaborativo y el ABP como método de enseñanza más usado, aunque en las variables analizadas la frecuencia de uso de las metodologías es alta en comparación de la preparación pertinente del docente.

Descriptores: Competencias del docente; formación de docentes; práctica pedagógica; aprendizaje activo. (Palabras tomadas del Tesauro UNESCO).

\begin{abstract}
The research aimed to analyze the use of active methodologies by university professors of medical sciences in order to generate meaningful learning in students. By means of a correlational descriptive type in a universe of 90 professors of the Medicine Career of the Catholic University of Cuenca. The results indicate that the frequency of the use of active methodologies in classes with the main difficulties observed in their students did not find a statistically significant relationship, while the variables of academic preparation in pedagogy in the teachers were verified, denoting higher values of those who did not they had such preparation, in the same way the use of Collaborative Work and ABP as the most used teaching method was outstanding, although in the analyzed variables the frequency of use of the methodologies is high compared to the pertinent teacher preparation.
\end{abstract}

Descriptors: Teacher qualifications; teacher education; teaching practice; activity learning. (Words taken from the UNESCO Thesaurus). 


\section{INTRODUCCIÓN}

La Ley Orgánica de Educación Superior (Asamblea Nacional Constituyente, 2018) en su Art. 13 refiere "incrementar y diversificar las oportunidades de actualización y perfeccionamiento profesional para los actores del sistema" (p.12) referente a la actuación de la docencia universitaria requiere constante actualización tanto en la rama profesionalizarte de la catedra, así como en pedagogía; a la vez, el Estatuto Orgánico de la Universidad Católica de Cuenca (Universidad Catolica de Cuenca, 2019) en su Art. 62 menciona "Los profesores titulares agregados o auxiliares deberán contar como mínimo con título de maestría afín al área en que ejercerán la catedra" (p.77). esto denota la ausencia en preparación pedagógica como requisito para ejercer la docencia universitaria.

El presente estudio pretende elucidar que en la enseñanza de las ciencias médicas en el ámbito universitario el aprendizaje al que llegan los estudiantes difiere de ser significativo, a la vez se sustente en aportes teóricos de (Rivadeneira Rodriguez, 2017), (Genes Diaz, Najera Polo, \& Monroy Toro, 2017), (Puga Peña \& Jaramillo Naranjo, 2015) mismos que en sus investigaciones realizadas en universidades del Ecuador demostraron que en la educación universitaria actual se necesita generar procesos de construcción del conocimiento, que dota a los estudiantes competencias reflexivas, críticas y autónomas. (Puga Peña \& Jaramillo Naranjo, 2015) en la Universidad Politécnica Salesiana de la Ciudad de Cuenca determino que el uso de metodologías activas de enseñanza genera importantes resultados en el aprendizaje para la resolución de problemas, así como la construcción del conocimiento y trabajo colaborativo (Erazo \& Narváez, 2020).

Desde la perspectiva de la educación universitaria el proceso enseñanza aprendizaje demanda especial atención a la interacción indispensable del docente y el alumno, dicha relación permite al estudiante construir su conocimiento, creando estudiantes con competencias críticas, reflexivas y autónomas. La pedagogía actual ha diseñado una serie de metodologías activas de la enseñanza que parten desde una perspectiva constructivista, cognitiva y experimental; desde cualquier mirada estas metodologías 
potencian la construcción del conocimiento, no solo trasmitir ideas fraccionadas de un método pedagógico, más bien enfocada en la creación del conocimiento (Puga Peña \& Jaramillo Naranjo, 2015).

La docencia de alta calidad, requiere una preparación continua de desarrollo profesional, las nuevas políticas de educación superior, buscan implementar en las aulas universitarias un cambio metodológico de enseñanza aprendizaje activo, que permita el desarrollo de competencias, centrándose en el aprendizaje autónomo del alumno de acuerdo a sus intereses. El objetivo de las metodologías es transformar el modelo tradicional en un proceso de enseñanza activo, interdisciplinario y participativo, que potencie la formación integral y la trasferencia de conocimiento (Gomez Hurtado, Carrasco Macias, \& Garcia Rodriguez, 2016).

La Carrera de Medicina de la Universidad Católica de Cuenca consta de 105 médicos especialistas que imparten docencia a 2062 estudiantes en todos sus niveles de preparación, la experticia profesional así como sus especialidades fueron vinculantes en el proceso de selección de docentes, a la vez en el ejercicio de la cátedra universitaria, se requiere conocimientos en pedagogía así como en estrategias activas de enseñanza aprendizaje, a fin de proporcionar al alumno un conocimiento que perdure en su preparación. Las ciencias médicas exigen reconocer la importancia del docente universitario y su didáctica; el hablar de analfabetismo pedagógico en las aulas universitarias conlleva a descuidar los diferentes modos de aprender de cada persona, sus competencias personales y la utilización de sus sentidos dirigidos hacia el aprendizaje, información que las metodologías activas dotan al docente para identificarlas (Arroyo-Carrera, et al., 2020).

Lo planteado dirige la atención no a conceptualizar las metodologías activas de enseñanza en la didáctica universitaria en el proceso de enseñanza de una cátedra en específico, más bien en develar el impacto que el desconocimiento de las mismas provoca en la generación del conocimiento a una educación memorizaría, poco objetiva, de evocación limitada únicamente para dar respuesta a una evaluación, sin mencionar 
que cada persona desarrollo aptitudes específicas de aprendizaje y se acopla a más a un método que a otro impuesto por el docente (González-González, et al., 2020).

El propósito de transformar la enseñanza tradicional en un aprendizaje activo, el fin esencial del uso de las metodologías activas es que un estudiante genere conocimientos y proporcionar a los alumnos la inclusión de técnicas innovadoras que favorezcan el aprendizaje activo y pueda relacionarlos con la cotidianidad de su contexto, facilitando la solución de conflictos a la vez que despierta su creatividad e ingenio (Puga Peña \& Jaramillo Naranjo, 2015).

En la ejecución del método de enseñanza innovador, en la actualidad la educación superior busca proyectarse a la interacción del maestro y el alumno integrando varias disciplinas, proceso en el que el docente organiza las actividades relacionadas al objeto de estudio en la búsqueda de soluciones a problemas reales, (Argandoña-Mendoza, et al., 2020), esto produce que el estudiante asimila el contenido del proceso de enseñanza; dicha propuesta fortalece el cambio del paradigma tradicional, sosteniendo como eje central la sujeción de aprendizaje participativo, contemplando problemas contextualizados desde el inicio de la enseñanza (Puga Peña \& Jaramillo Naranjo, 2015) Según (Rodriguez, Ramirez, \& Fernandez, 2017) mencionan "La literatura muestra muchas variantes de esta metodología tales como el ABP o el aprendizaje basado en problemas, MDC o método de casos, ABP y o aprendizaje basado en proyectos y el aprendizaje colaborativo" (p. 4). esto refiere las diferentes formas de abordaje que utiliza las metodologías activas en la enseñanza actual.

Siguiendo a (Remache Irure \& Belletich, 2015) en las ciencias médicas, como en cualquier disciplina el uso del ABP como método de enseñanza utiliza la construcción del conocimiento como principio instructivo, sujeta a la investigación - diagnostica de la percepción del docente y del alumno en relación a la solución de problemas, la efectividad del método esté ligado a mecanismos de repetición que involucra al alumno a desarrollar capacidades, actitudes, habilidades y valores, a la vez motiva al docente a ser un guía que aliente al estudiante a descubrir por cuenta propia y sentirse satisfecho por lo 
adquirido (García-Cedeño, et al., 2020).

\section{Aprendizaje significativo}

En coherencia con lo planteado por Ausubel en 1963 en su trabajo sobre psicología del aprendizaje verbal significativo, este diferencia la enseñanza y el aprendizaje y señala al aprendizaje significativo como tarea esencial de la educación, relacionada con los conocimientos adquiridos previo a la introyección de nueva información; es primordial restar importancia a una adquisición memorística o repetitiva, ya que un conocimiento para ser significativo solo sería posible si exigiera una relación entre el nuevo conocimiento y el previo que posee el educando. Consecuentemente el aprendizaje significativo es estudiado y puesto en práctica desde los diferentes enfoques de modelos pedagógicos que han intentado comprenderlo (Olaya \& Ramirez, 2015)

Posición contraria la de (Olaya \& Ramirez, 2015) "Io significativo es el aprendizaje, no la enseñanza o método mediante el cual se da la enseñanza". En consecuencia, lo significativo son los aprendizajes comprensibles, reorganizados y relevantes que el pensamiento adquiere por repetición y descubrimiento, determinado considerablemente por el contexto en el que se desarrolle (Quevedo-Álava, et al., 2020).

Dentro del planteamiento tradicionalista de escuela, la participación docente es primordial en el aprendizaje del estudiante, dado que es el encargado es delimitar lo que el alumno aprende y de qué manera lo hace. Desde una mirada innovadora constructivista el estudiante universitario centra su aprendizaje en la construcción como proceso a partir de la interacción, limitándose no solo con el docente, sino con el ambiente y el grupo de trabajo; enfoque que promueve la creación de grupos y comunidades de aprendizaje, en esta perspectiva la enseñanza se entiende no como la trasferencia de información al estudiante, más bien, motiva el desarrollo de competencias para construir y reconstruir conocimientos en respuesta a sus necesidades, (Rodriguez, Ramirez, \& Fernandez, 2017). 
Por ello la importancia de tomas como estrategia de fortalecimiento del ejercicio docente al trabajo colaborativo como metodología activa. La interacción de los participantes en el proceso de aprendizaje insta al grupo a compartir las tareas y aportar en común una misma idea, el trabajo colaborativo es visto como un proceso en el que cada sujeto aprende más de lo que podría hacerlo por cuenta propia, ya que una actividad relacionada al aprendizaje realizado en grupo de forma colaborativa, genera un resultado de mayor relevancia que la suma de trabajos individuales (Saltos-Cedeño, et al., 2020).

Al definir competencia se hace alusión a una realización eficiente y satisfactoria de actividades meta cognitivas enfocadas a responder a demandas complejas de un contexto determinado, relacionadas por la movilización de destrezas, conocimiento, practica, actitudes comportamentales, valores y emociones; la adquisición de competencias de aprendizaje tiene mucha relación con los estilos de aprendizaje de cada sujeto que de acuerdo a su formación y experiencia integra y moviliza sus recursos disponibles en la resolución de una problemática actual (Reyes Roa, 2017).

El desarrollo de estas competencias a más de las capacidades adquiridas previamente por el estudiante se refuerza por el uso de metodologías activas del aprendizaje, entre las que se cita el aprendizaje basado en problemas, realización de prácticas contextualizadas, trabajo colaborativo, el aprendizaje en red y el aprendizaje basado en la experiencia que inciden en la generación del aprendizaje autónomo. (Perez Dueñas \& Antoli Cabrera, 2016) trata al trabajo colaborativo como un enfoque experimental que el alumno desarrollo sobre apreciaciones de diferentes perspectivas, detallando sus conocimientos a través de debates, argumentaciones y compartiendo ideas con sus semejantes, así como recibiendo retroalimentación y feedback de los demás. Sin duda alguna el alumno reflexiona sobre su propio aprendizaje favoreciendo su posterior manejo de conflictos tal como se generan en contextos laborales reales (Vélez-Loor, et al, 2020). La presente investigación tiene como objetivo analizar el uso de metodologías activas por parte de docentes universitarios de las ciencias médicas con la finalidad de generar un aprendizaje significativo en los estudiantes. 


\section{MÉTODO}

La investigación se operacionalizó en función de un tipo descriptiva correlacional no experimental transeccional con análisis de Chi-cuadrado de Pearson (Erazo-Álvarez \& Narváez-Zurita, 2020), se aplicó una encuesta y cuestionario en escalamiento en Likert a un universo de 90 docentes de la Carrera de Medicina de la Universidad Católica de Cuenca. Acotándose que se generó una sección descriptiva de análisis de información con la finalidad de complementar el Chi-cuadrado de Pearson.

\section{RESULTADOS}

De los resultado obtenidos se puede inferir entre las diferentes problematicas observadas en este estudio, los principales hallazgos se obtuvieron al relacionar la frecuencia de la utilizacion de metodologias activas de la enseñanza en el aula clase con las principales dificultadas percibidas por los docentes en su funcion, para ello se aplico la prueba de Chi-cuadrado de Pearson donde se evidenció la siguiente ralacion: de 90 docentes universitarios encuestados 84 siempre y frecuentemente hace uso de metodologias activas de la enseñana en sus clases, donde 26 de ellos encuentro como dificultad la falta de motivacion en los alumnos y 30 considera la falta de atencion como la dificultad frecuente. Al aplicar la prueba de Chi-cuadrado se obtiene un valor de significacion asistotica bilateral de 0,349 , resultado que no es significativamente estadistico para relaacionar tales variables. 


\section{Tabla 1}

Tabla cruzada uso de metodologías activas de enseñanza*Dificultades al realizar docencia

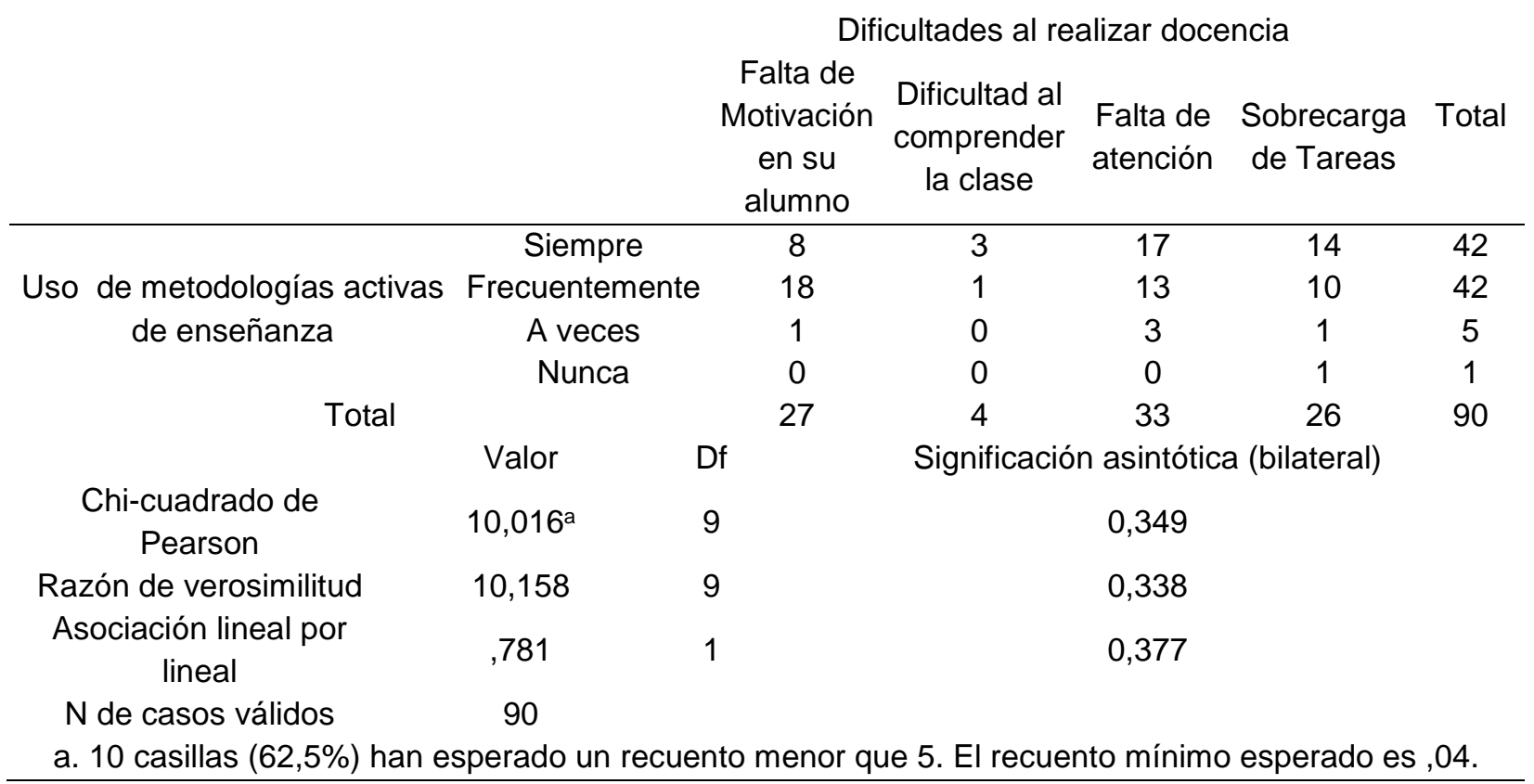

\section{Fuente: Elaboración Propia}

A la luz de los hallazgos arrojados al analizar la frecuencia de la formación académica que poseen los docentes universitarios en su preparación profesional, se observó que un $44.4 \%$ si presenta preparación en pedagogía, en cambio el $54.4 \%$ de los encuestados no tiene preparación en pedagogía. Este resultado afianza el objetivo de estudio planteado en la investigación, al tratar el analfabetismo pedagógico en la docencia universitaria. 


\section{Tabla 2}

Formación Académica

\begin{tabular}{|c|c|c|c|c|c|}
\hline & & Frecuencia & Porcentaje & Porcentaje válido & $\begin{array}{l}\text { Porcentaje } \\
\text { acumulado }\end{array}$ \\
\hline \multirow{4}{*}{ Válido } & $\mathrm{Si}$ & 40 & 43,5 & 44,4 & 44,4 \\
\hline & No & 49 & 53,3 & 54,4 & 98,9 \\
\hline & 3 & 1 & 1,1 & 1,1 & 100,0 \\
\hline & Total & 90 & 97,8 & 100,0 & \\
\hline \multirow{2}{*}{ Perdidos } & Sistema & 2 & 2,2 & & \\
\hline & & 92 & 100,0 & & \\
\hline
\end{tabular}

Fuente: Elaboración propia

Se analizó la variable de frecuencia en la utilización de metodologías activas en el aula de clase, resultando que de la totalidad de encuestados el $32.2 \%$ refiere que siempre usa metodologías activas en su enseñanza, a la vez el 57.8\% frecuentemente hace dicho uso, así como el 8.9\% manifiesta que a veces utiliza las metodologías como vehículo para su enseñanza, y tan solo un $1.1 \%$ de docentes experimenta que nunca ha hecho uso de las metodologías activas en su función docente.

\section{Tabla 3}

Frecuencia de uso metodologías activas

\begin{tabular}{cccccc} 
& & Frecuencia & Porcentaje & $\begin{array}{c}\text { Porcentaje } \\
\text { válido }\end{array}$ & $\begin{array}{c}\text { Porcentaje } \\
\text { acumulado }\end{array}$ \\
\hline \multirow{4}{*}{ Válido } & Siempre & 29 & 31,5 & 32,2 & 32,2 \\
& Frecuentemente & 52 & 56,5 & 57,8 & 90,0 \\
& A veces & 8 & 8,7 & 8,9 & 98,9 \\
\multirow{4}{*}{ Perdidos } & Nunca & 1 & 1,1 & 1,1 & 100,0 \\
& Total & 90 & 97,8 & 100,0 & \\
& Sistema & 2 & 2,2 & & \\
& Total & 92 & 100,0 & &
\end{tabular}

Fuente: Elaboración propia

Las metodologías activas de enseñanza utilizadas con frecuencia como estrategias pedagógicas se consideraron como variable sustantiva, al analizar la frecuencia de su uso en la docencia; se observó como principales hallazgos que el $45.6 \%$ usa el Trabajo Colaborativo como su metodología activa, el 35.6\% considera al Aprendizaje Basado en 
Problemas como su estrategia más utilizada, un $13.3 \%$ de ellos utiliza el Aula invertida o Flipped Classroom y el $5.6 \%$ de docentes encuestados utiliza el Aprendizaje Basado en Proyectos como estrategia de enseñanza.

\section{Tabla 4}

Estrategias y recursos

\begin{tabular}{clcccc} 
& Frecuencia & Porcentaje & $\begin{array}{c}\text { Porcentaje } \\
\text { válido }\end{array}$ & $\begin{array}{c}\text { Porcentaje } \\
\text { acumulado }\end{array}$ \\
\hline \multirow{6}{*}{ Válido } & Aprendizaje Basado en Proyectos & 5 & 5,4 & 5,6 & 5,6 \\
& Trabajo Colaborativo & 41 & 44,6 & 45,6 & 51,1 \\
& Flipped Classroom o Aula invertida & 12 & 13,0 & 13,3 & 64,4 \\
& Aprendizaje Basado en Problemas & 32 & 34,8 & 35,6 & 100,0 \\
& Total & 90 & 97,8 & 100,0 & \\
Perdidos & Sistema & 2 & 2,2 & & \\
& \multicolumn{1}{c}{ Total } & 92 & 100,0 & & \\
\hline
\end{tabular}

Fuente: Elaboración propia

Al relacionar nuestras variables del uso de metodologías activas de la enseñanza con las principales dificultades encontradas por los docentes en sus aulas de clase, mediante la fórmula de Chi-cuadrado se obtuvo como resultado una significación asintótica estadísticamente no significativa para la investigación, queriendo demostrar que al momento en que los docentes hacen uso de las metodologías activas de la enseñanza lo realizan de manera equivoca, probablemente desconociendo el proceso sintónico de las metodologías.

Así lo demuestra (Sandi Delgado \& Cruz Alvarado, 2016) en su investigación donde propuso el uso de metodologías de enseñanza como estrategia para innovar la educación superior, obteniendo como resultados que la fortaleza del uso de metodologías radica en la variedad de actividades propias del proceso metodológico, refiriendo que las mismas deben estar secuencialmente operada en función a los contenido de aprendizaje, las ideas de los estudiantes y presentar tares especificas contextualizada enfocado en un trabajo grupal, individual y posibilitando la interacción del medio, esta discusión evidencia que las dificultades encontradas por los docentes encuestados en esta investigación, 
tales como la falta de motivación y la falta de atención se deben a la operación asintótica y carente de metodologías propias para fomentar el interés en los estudiantes.

Por otro lado, (Faraba Garzon, 2016) menciona en su investigación sobre docencia universitaria en el Ecuador, al medir la preparación pedagógica como un desafío en la innovación de la educación superior, llega a la conclusión que en el 2015 la formación académica que tuvieron los docentes universitarios no se ha desarrollado con el interés que prima tener, refiriendo que la oferta académica existente en el Ecuador y fuera de él no alcanza a satisfacer las demandas que el sector necesita para su funcionamiento, cita con especial atención que las legitimidad existente en las normativas públicas no establece como obligatoriedad que el docente necesite en su preparación, destacando que en la función docente en la educación superior tiene muchas importancia la preparación académica en pedagogía puesto que este requisito supera ampliamente el ejercicio de una catedra. En concordancia con nuestros resultados donde obtuvimos de 90 docentes encuestados el 53.3\%no registra preparación académica en pedagogía y el $43.5 \%$ si la tiene, este dato denota y justifica el objetivo de estudio al referir el analfabetismo pedagógico como una problemática en la enseñanza de la Educación Superior.

En la Unidad Educativa Reino de Inglaterra, Ecuador, (Cano de la Cruz, Aguiar Monar, \& Mendoza Roman, 2019) desarrollaron una investigación a docentes, cuyo objetivo fue medir el nivel de uso de metodologías activas en su clase, obteniendo como resultado que un $35.71 \%$ hace uso frecuente de las metodologías activas, a la vez el $7.14 \%$ no utiliza metodologías como camino a la educación. A la relación con nuestra investigación encontramos relación, que el $32.2 \%$ siempre utiliza metodologías activas, coincide con la investigación de Cano de la cruz y colaboradores; no obstante, el $8.9 \%$ de encuestados en este estudio determino que a veces y nunca hacen uso de metodologías en su enseñanza, estrecha relación de discusión con el estudio en cuestión. 


\section{PROPUESTA}

El uso de metodologías activas en la docencia universitaria ha demandado somera atención para lograr un aprendizaje significado en educandos de las ciencias médicas, a la luz de los resultados obtenidos en este estudio se ha dado respuesta a nuestra objeto de estudio, al considerar la escasa preparación pedagógica en docentes así como su nivel de uso de metodologías activas, planteamos como propuesta ejecutar un plan de acción basado en el esquema de implementar, ejecutar, fortalecer y actuar.

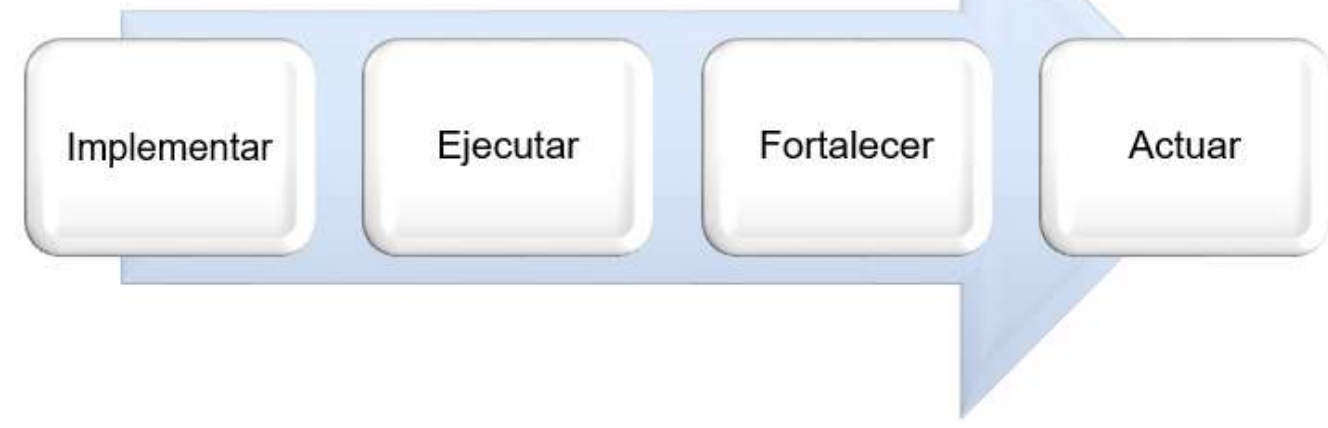

Figura 1. Propuesta de capacitación docente. Fuente: Elaboración propia

Implementar.- La necesidad de capacitación docente en metodologías y estrategias activas de la enseñanza con la finalidad de cubrir la demanda de catedráticos a nivel local y regional, a través de planes de posgrado o capacitaciones certificadas que doten al docente estrategias no solo en el conocimiento de la teoría de la catedra, sino en el método adecuado para trasferir este conocimiento a sus alumnos, donde las dificultades encontradas como la atención o motivación del alumno se vean contrapuestas por la interacción del estudiante y su proceso de aprendizaje, sin descuidar el rol docente de facilitador del aprendizaje con didáctica secuencial, participativa, ordenada y sujeta a un proceso comprobado por su eficacia. 
Ejecutar. - Aplicar lo aprendido en los planes de posgrado sobre el uso de metodologías activas en sus estudiantes, a fin de que el estudio de las ciencias médicas llegue a un aprendizaje significativo y no momentáneo limitado a una evaluación, hacer de un estudiante un profesional crítico y reflexivo sobre sus saberes, con capacidad resiliente frente a la cotidianidad de su carrera profesional.

Fortalecer. - Las Instituciones de Educación Superior deben implementar programas de capacitación continua en pedagogía, dirigida a sus docentes, con planes de innovación educativa sujetas a las demandas actuales de la educación y la cultura; fortalecer a sus docentes con nuevas estrategias garantiza el aprovechamiento académico de sus alumnos sea sobre el promedio, y así cumplir con el perfil de egreso de su propuesta académica.

Actuar. - La educación superior actual es imperativa en sus alcances y necesidades, poner en marcha una modificación sustantiva de la planificación curricular en la carrera médica, donde se dé énfasis no solo al contenido sino al camino para llegar a él, la sobrecarga psicológica que provoca el estudio de la medicina sumado a un método anticuado y ortodoxo aplicado en el aula de clase tiene repercusiones en nuestros profesionales de la salud. Modificar el método de enseñanza garantiza calidad.

\section{CONCLUSIONES}

A la luz de los principales hallazgos resultantes de esta investigación, se pudo responder el objeto de estudio al considerar el analfabetismo pedagógico en la docencia universitaria como eslabón fundamental en el aprendizaje significativo en las ciencias médicas, detallaremos las principales conclusiones obtenidos.

Se comparó la variable de dificultades encontradas por los docentes en sus alumnos con el uso de metodologías activas, no encontrándose relación a la frecuencia elevada de uso de metodologías, esto se explica mejor, analizando que si bien es cierto gran parte de docentes aplica estrategias de enseñanza en sus clases, lo desarrollan de una manera no secuencial o podría decirse carente de método, es por ello que en el estudio refleja 
índices altos de problemas de concentración y atención de sus alumnos, ya que una metodologías activa de la enseñanza está diseñada para contrarrestar la falta de atención y motivación de los alumnos.

La formación académica en pedagogía de docentes universitarios es primordial para un aprendizaje significativo, sujeto a las demandas de la sociedad y la tecnología, en nuestra investigación se evidencio la marcada necesidad de capacitación docente que muestra la educación superior, al superar más del $50 \%$ de falta de preparación pedagógica en docentes de educación superior.

Al medir la frecuencia del uso de metodologías en las ciencias médicas pudimos encontrar que la mayoría de docentes encuestados hacen uso de metodologías activas, fortaleciendo los resultados anteriores y tan solo una minoría no las usa, este dato responde a las exigencias de excelencia que demanda la institución superior de estudio. En el ejercicio de la docencia nos interesamos en definir las metodologías más usadas por los docentes, resaltando el Aprendizaje Colaborativo y el Aprendizaje Basado en Problemas como las estrategias más usadas; se interpreta esta frecuencia por las facilidades que presta el trabajo colaborativo en un grupo de estudio y el ABP como método de constante capacitación.

\section{FINANCIAMIENTO}

No monetario

\section{AGRADECIMIENTOS}

A los docentes de la Carrera de Medicina de la Universidad Católica de Cuenca, por apoyar esta investigación. 


\section{REFERENCIAS}

Asamblea Nacional Constituyente. (2018). Ley Organica de Educacion Superior[Organic Law of Higher Education]. Quito. Obtenido de https://n9.cl/gotv

Argandoña-Mendoza, M., García-Mejía, R., Ayón-Parrales, E., \& Zambrano-Zambrano, Y. (2020). Investigación e innovación educativa: Reto escolar por COVID-19 en el Ecuador. KPISTEME KOINONIA, 3(5), 162-182. http://dx.doi.org/10.35381/e.k.v3i5.726

Arroyo-Carrera, E., Loor-Santos, M., Mendoza-Mera, J., \& Solorzano-Zambrano, M. (2020). Gestión de aprendizaje creativo mediante la Herramienta Powtoon en estudiantes de lengua y literatura. [Creative learning management through the Powtoon Tool in language and literature students]. EPISTEME KOINONIA, 3(5), 251-267. http://dx.doi.org/10.35381/e.k.v3i5.775

Cano de la Cruz, Y., Aguiar Monar, J. C., \& Mendoza Roman, M. C. (2019). Metodologías activas: una necesidad en la Unidad Educativa Reino de Inglaterra[Active methodologies: a necessity in the Kingdom of England Educational Unit]. Revista Educación, 43(2). doi:10.15517/REVEDU.V4312.29094

Erazo, J. C., \& Narváez, C. I. (2020). La gestión del capital intelectual y su impacto en la efectividad organizacional de la industria de cuero y calzado en la Provincia de Tungurahua - Ecuador [The management of intellectual capital and its impact on the ] Recuperado de: https://n9.cl/52li. Revista Espacios, 254-271.

Erazo-Álvarez, J. C., \& Narváez-Zurita, C. I. (2020). Medición y gestión del capital intelectual en la industria del cuero - calzado en Ecuador. [Measurement and management of intellectual capital in the leather industry - footwear in Ecuador]. Revista Arbitrada Interdisciplinaria Koinonía, 437-467. doi:http://dx.doi.org/10.35381/r.k.v5i9.662

Faraba Garzon, E. (2016). La formación y el ejercicio de la docencia universitaria en Ecuador. Desafíos[The training and exercise of university teaching in Ecuador. Challenges]. ALTERIDAD. Revista de Educación, 11(2), 170-181. Obtenido de https://n9.cl/rhwy9

García-Cedeño, G., Vélez-Loor, M., Franco-Zambrano, C., \& Ormaza-Bermello, M. (2020). Educación por competencias: Un reordenamiento curricular durante emergencia escolar por COVID-19. [Competency Education: A Curricular Rearrangement During School Emergency by COVID-19]. EPISTEME KOINONIA, 3(5), 221-235. http://dx.doi.org/10.35381/e.k.v3i5.770 
Genes Diaz, J., Najera Polo, F., \& Monroy Toro, S. (2017). Metodologias activas para la solucion de problemas al enseñar matematicas financieras[Active methodologies for solving problems when teaching financial mathematics]. Omnia, 44-58. Obtenido de https://n9.cl/t1am

Gomez Hurtado, I., Carrasco Macias, M. J., \& Garcia Rodriguez, M. d. (2016). Metodologias activas para la enseñanza plurilingue en estudiantes universitarios[Active methodologies for multilingual teaching in university students]. Magis. Revista Internacional de Investigacion en Educacion, 173-192. Obtenido de https://n9.cl/4bgfx

González-González, D., García-Herrera, D., Cabrera-Berrezueta, L., \& Erazo-Álvarez, J. (2020). Herramientas tecnológicas aplicadas por los docentes durante la emergencia sanitaria COVID-19. [Technological tools applied by teachers during the health emergency COVID-19]. Revista Arbitrada Interdisciplinaria Koinonía, 5(1), 332-350. http://dx.doi.org/10.35381/r.k.v5i1.786

Olaya, A., \& Ramirez, J. (2015). Tras la huella del aprendizaje significativo, lo alternativo $y$ la innovacion en el saber y la practica pegagogica [In the footsteps of meaningful learning, the alternative and the innovation in pedagogical knowledge and practice]. Revista Cientifica Guillermo de Ockham, 117-125. Obtenido de https://n9.cl/unyfg

Perez Dueñas, C., \& Antoli Cabrera, A. (2016). Desarrollar competencias en la educacion superior con trabajo autonomo y de investigacion[Develop skills in higher education with autonomous work and research]. Opcion, 238-253. Obtenido de https://n9.cl/xzl4

Puga Peña, I. A., \& Jaramillo Naranjo, L. M. (2015). Metodologias activas de la construccion del conocimiento matermatico[Active methodologies for the construction of mathematical knowledge]. Sophia, 291-314. Obtenido de https://n9.cl/zlcy

Quevedo-Álava, R., Corrales-Moreno, L., Palma-Delgado, G., \& Mendoza-Suárez, G. (2020). Psicopedagogía y TIC en período de COVID-19. Una reflexión para el aprendizaje significativo. [Psychopedagogy and ICT in the COVID-19 period. A Reflection for Meaningful Learning]. EPISTEME KOINONIA, 3(5), 202-220. http://dx.doi.org/10.35381/e.k.v3i5.769 
Remache Irure, A., \& Belletich, O. (2015). El Metodo de aprendizaje basado en proyectos $(A B P)$ en contxtos educativos rurales y socialmente desfavorecidos en la educacion infantil[The Project-Based Learning Method (ABP) in rural and socially disadvantaged educational contexts in early childhood ed]. Perspectiva Educacional, Formacion de Profesores, 90-109. Obtenido de https://n9.cl/odgd

Reyes Roa, M. L. (2017). Desarrollo de la competencia de aprendizaje autonomo en estudiantes de Pedagogia en un modelo educativo basado en competencias[Development of autonomous learning competence in Pedagogy students in a competency-based educational model]. REXE. Revista de Estudios y Experiencias en Educacion, 67-82. Obtenido de https://n9.cl/g49d

Rivadeneira Rodriguez, E. M. (2017). Competencias didacticas- pedagogicas del docente, en la trasnformacion del estudiante universitario[Didactic-pedagogical competences of the teacher, in the transformation of the university student]. Orbis, Revista Cientifica Ciencias Humanas, 13(37), 41-55. Obtenido de https://n9.cl/y0t8

Rodriguez, A. B., Ramirez, L. J., \& Fernandez, W. (2017). Metodologias Activas para Alcanzar el Comprender[Active Methodologies to Achieve Understanding]. Formacion Universitaria, 79-88. Obtenido de https://n9.cl/4cqs

Sandi Delgado, J. C., \& Cruz Alvarado, M. A. (2016). Propuesta metodologica de enseñanza y aprendizaje para innovar la educacion superior[Teaching and learning methodological proposal to innovate higher education]. InterSedes(36), 238. Obtenido de https://n9.cl/v8tu

Saltos-Cedeño, A., Vallejo-Valdivieso, P., \& Moya-Martínez, M. (2020). Innovación en educación matemática de básica superior durante el confinamiento por COVID19. [Innovation in higher basic mathematics education during confinement by COVID-19]. KPISTEME KOINONIA, $\quad 3(5), \quad 142-161$. http://dx.doi.org/10.35381/e.k.v3i5.723

Universidad Catolica de Cuenca. (2019). Estatuto Organico de la Universidad Catolica de Cuenca[Organic Statute of the Catholic University of Cuenca]. Cuenca: Repositorio Institucional de Documentacion Abierta. Obtenido de https://n9.cl/h7vgc

Vélez-Loor, M., Vallejo-Valdivieso, P., \& Moya-Martínez, M. (2020). Recursos didácticos virtuales en proyectos de ciencias naturales en período de confinamiento por COVID-19. [Virtual teaching resources in natural science projects in confinement period by COVID-19]. EPISTEME KOINONIA, 3(5), 183-201. http://dx.doi.org/10.35381/e.k.v3i5.760 
EPISTEME KOINONIA

Revista Electrónica de Ciencias de la Educación, Humanidades, Artes y Bellas Artes

Año III. Vol III. Nº6. Julio - Diciembre 2020

Hecho el depósito de Ley: FA2018000022

ISSN: 2665-0282

FUNDACIÓN KOINONIA (F.K).

Santa Ana de Coro, Venezuela

Luis Miguel Ormaza Ulloa; Darwin Gabriel Garcia-Herrera; Juan Carlos Erazo-Álvarez; Cecilia Ivonne Narváez Zurita

@2020 por el autor. Este artículo es de acceso abierto y distribuido según los términos y condiciones de la licencia Creative Commons Atribución-NoComercial-Compartirlgual 4.0 Internacional (CC BY-NC-SA 4.0)

(https://creativecommons.org/licenses/by-nc-sa/4.0/). 